\title{
Inner Products and Orthogonality in Color Recording Filter Design
}

\author{
Poorvi L. Vora
}

\begin{abstract}
We formalize ideas of orthogonality and inner products implicit in the development of a number of figures of merit (FOM, [1]) of color recording filters. We show that, in negligible measurement noise, the data dependence of each FOM based on linear color correction is equivalent to a choice of inner product (and hence of orthogonality). Further, we show that optimal sensors with respect to noise sensitivity are simply defined as orthogonal with respect to this inner product. We also develop the idea of a generalized $Q$-factor by generalizing the Euclidean inner product to include all inner products. Simulations demonstrate the utility of our analytical results.
\end{abstract}

Index Terms-Color, digital cameras, orthogonality, recording filters, scanning filters.

\section{INTRODUCTION}

$\mathbf{E}$ LECTRONIC image capture devices like scanners and digital cameras use charge-coupled device (CCD) or active pixel sensor (APS) technology for sensor fabrication. The color capabilities of these devices come about from the color filters that are used with the sensors, and there has been considerable interest and literature on the design of optimal color filters from the point of view of color fidelity [2]-[7], [1] and robustness [6], [8]-[10]. There is a wide range of criteria for color fidelity-varying in colorimetric accuracy, parameters and computational complexity. The common aspect of most of the criteria is that they may be expressed in terms of inner products, as we will show in Section III. The existing investigations into robustness of a recording filter set [6], [8]-[10] are based on specific color fidelity criteria.

This paper poses the problem of the accuracy of color recording filters in terms of inner products and orthogonality-exploiting the common aspect of most existing performance criteria. This allows a common framework for noise analysis. We define an inner product using data-dependence in the form of preferential weighting of errors in directions where most of the energy of the data set is concentrated. We show that Neugebauer's $Q$-factor can be extended using the generalized inner product. We illustrate the use of the generalized inner product, the induced generalized orthogonality and generalized $Q$-factor by addressing the problems of the characterization of color filters with respect to color fidelity and noise sensitivity.

The problem of color fidelity has been addressed satisfactorily by a number of researchers, and we show that the inner

Manuscript received November 13, 1998; revised June 14, 2000. The associate editor coordinating the review of this manuscript and approving it for publication was Prof. Glenn Healey.

The author is with Hewlett-Packard Laboratories, Corvallis, OR 97330 USA (e-mail: poorvi@hpl.hp.com).

Publisher Item Identifier S 1057-7149(01)01657-8. product provides a single framework for similar approaches. We generalize the fundamental error, based on orthogonal directions in the human visual subspace (HVSS), to other quadratic error measures in the target color space. The FOMs of Sharma and Trussell [1] and Wolski et al. [6] for negligible noise, Neugebauer [2], Vora and Trussell, $(\nu),[3]$ and Finlayson [7], are all choices of an inner product, and hence choices of "orthogonality." The purpose of this paper is to demonstrate the use of inner products in the simplification of optimality expressions for color recording filters. The rich mathematical results available for inner products may be used to produce other results in the future.

We address the problem of noise sensitivity in detail and show that optimal filters with respect to noise sensitivity are those that are orthonormal with respect to the generalized inner product. Vrhel and Trussell [4] show that optimal filters with respect to sensitivity to filter fabrication errors also satisfy the same criterion. We present the result here in a simpler form than they do, in terms of inner products and orthonormality. The initial part of the paper, which deals with inner products, is essential to an understanding of the latter part, which deals with orthogonality.

The paper is organized as follows. We establish background in Section II. In Section III, we define the tools we use in the rest of the paper-generalized inner products, orthogonality, projection operators and $Q$-factors. In Section IV, we describe the use of the tools in studying colorimetric accuracy-in particular, we describe the specific translations from color space and data statistics to inner products. In this section we also show how the measures of [1]-[3], [6], correspond to choices of inner products. We also show that these measures may be expressed as a weighted sum of generalized $Q$-factors of preferred directions in the target space. We present the use of our tools for noise analysis in Section V. In particular, we show why orthogonality is important for optimality of recording filters. Simulation results demonstrating the usefulness of the framework and the noise sensitivity results are in Section VI. A summary of the major results of the paper is presented in Section VII.

\section{PRELIMINARIES}

The notation in this paper follows that of Trussell [11], Vora and Trussell [3], [9] and Sharma and Trussell [1]. A list of the notation used may be found in the Appendix. Symbols are also defined where they are first used. Filter transmissivities, spectral reflectance functions, radiant illuminant spectral distributions, the CIE matching functions [12, pp. 130-143] and all other functions of wavelength are assumed to be represented by $N$ samples in the visual range. The theoretical results presented 
here and the ideas used are independent of the sampling rate. The simulations have been performed for $N=31$.

The common problem in color scanning, digital photography, and color correction is the design of filters to obtain the values

$$
\mathbf{t}=\mathbf{V}^{T} \mathbf{f}
$$

where $\mathrm{f}$ is an $N$-vector representing the visual stimulus. The matrix $\mathbf{V}$ consists of $s$ columns, $\mathbf{V}=\left[\mathbf{v}_{1}, \mathbf{v}_{2}, \cdots \mathbf{v}_{s}\right]$. The columns of $\mathbf{V}$ represent the combined effect of the CIE matching functions and a viewing illuminant in the case of color scanning and digital photography, or the combined effect of the CIE matching functions and many different viewing illuminants in the case of color correction. The vector $\mathbf{t}$ may be referred to as the $s$-stimulus vector.

If the columns of matrix $\mathbf{A}$ represent the CIE color matching functions, and the diagonal matrix $\mathbf{L}$ represents the viewing illuminant, the matrix product LA represents the color matching functions for the viewing illuminant represented by $\mathbf{L}$ and is denoted $\mathbf{A}_{L}$. The vector $\mathbf{t}$ is the tristimulus vector and $s=3$ when $\mathbf{V}=\mathbf{A}_{L}$.

This formulation allows the linear model ideas of [2], [3], [11], [13]-[15] to be extended to define color fidelity criteria of sets of recording filters used for multi-band spectral measurements, even when the measurements are not those of the CIE tristimulus values [16]. The designed filters do not need to replicate the columns of $\mathbf{V}$, and it is sufficient to obtain measurements from which the values $\mathbf{V}^{T} \mathbf{f}$ may be determined through a linear transformation [3], [11], [13]. The properties of the linear transformation determine the noise amplification inherent in the procedure, and this is discussed in detail in Section V.

If the matrix $\mathbf{M}$ represents the set of recording filters-either scanning filters or filters on a camera-and $\mathbf{H}$ is a diagonal matrix representing the combined effect of the recording illuminant, the optical path and the sensor, the matrix HM, denoted $\mathbf{M}_{H}$, represents the effective recording system. Its output is $\mathbf{g}=\mathbf{M}_{H}^{T} \mathbf{f}+\mathbf{n}$, where $\mathbf{n}$ represents measurement noise. The $s$-stimulus values, $\mathbf{t}$, may be estimated from the measurements $\mathrm{g}$ using standard linear methods such as those used to solve sets of simultaneous equations. The estimation procedure involves inverting the effect of the recording system, estimating $\mathbf{f}$, and thus estimating the inner product of $f$ with the columns of $\mathbf{V}$.

The linear minimum mean square error (LMMSE) estimate of the $s$-stimulus values of zero-mean signal $\mathbf{f}$ in the presence of zero-mean signal-uncorrelated measurement noise is [4], [8]

$$
\hat{\mathbf{t}}=\mathbf{V}^{T} \mathbf{R} \mathbf{M}_{H}\left(\mathbf{M}_{H}^{T} \mathbf{R} \mathbf{M}_{H}+\mathbf{R}_{n}\right)^{-}\left(\mathbf{M}_{H}^{T} \mathbf{f}+\mathbf{n}\right)
$$

where $\mathbf{R}=E\left[\mathrm{ff}^{T}\right]$ and $\mathbf{R}_{n}=E\left[\mathbf{n n}^{T}\right]$ are the sample correlation matrices of the data and the noise respectively. The incorporation of nonzero signal and noise means does not change the basic results of the analysis.

The correction matrix is the linear transformation used to obtain the estimate from the measurements

$$
\mathbf{B}=\mathbf{V}^{T} \mathbf{R M}_{H}\left(\mathbf{M}_{H}^{T} \mathbf{R} \mathbf{M}_{H}+\mathbf{R}_{n}\right)^{-} .
$$

The properties of the matrix $\mathbf{B}$ determine the noise amplification properties of the procedure of determining the target $s$-stimulus values using the effective recording system $\mathbf{M}_{H}$.

The error in estimating the $s$-stimulus values may be calculated in many ways. Linear models have been largely successful in explaining the color responses of the sensor and filter combination [17]-[19], though errors perceived by the human visual system are far from linear. Commonly used error measures for the color reproduction of patches include the mean-square error in a linear transformation of the CIE tristimulus space and the mean-square error in the perceptually uniform CIELAB space $[12$, p. 166]. We discuss these errors in more detail in the rest of this section.

A common instance where linear transformations of the tristimulus values are the target of measurements is when a color is to be reproduced on an additive display, like a CRT monitor. While the mean-square error in a linear transformation of CIE tristimulus space is not a good approximation of perceptual error, it provides a numerical estimate of colorimetric accuracy and may be manipulated with the use of simple mathematics. Hence it is commonly used for rough optimality estimates. Euclidean distance in a linear transformation of the $s$-stimulus space corresponds to a weighted Euclidean distance in the original space, and is a quadratic error measure because it is of the form $\sum \mathbf{e}_{i} w_{i j} \mathbf{e}_{j}$, where $\mathbf{e}$ symbolizes the error vector, and $w_{i j}$ the weights.

Let $\mathbf{C}$ denote the transformation from the $s$-stimulus space to a space in which Euclidean distance better represents perceptual error. When $\mathbf{C}$ is the identity, the error is measured as Euclidean distance in the space of the $s$-stimulus values. Hence, when the $s$-stimulus values are the CIE tristimulus values, and $\mathbf{C}=\mathbf{I}$ the above error is the mean square tristimulus error.

In some instances, it is useful to determine the error between fundamentals [14], i.e., the difference between the parts of a visual stimulus that do not lie in the "black space" of the human visual system. The fundamental, as defined in [14], is the projection of a visual stimulus onto the space spanned by the color matching functions (the HVSS). When the viewing illuminant is fixed and known and taken into consideration, the fundamental is the projection onto the human visual illuminant subspace (HVISS). We denote the projection operator onto the space spanned by the columns of matrix $\mathbf{V}$ as $P_{V}$, $P_{V}=\mathbf{V}\left(\mathbf{V}^{T} \mathbf{V}\right)^{-1} \mathbf{V}^{T}$. When $\mathbf{C}$ corresponds to determining $P_{V}$, i.e., when $\mathbf{C}=\mathbf{V}\left(\mathbf{V}^{T} \mathbf{V}\right)^{-1}$, the error is measured as the Euclidean distance between fundamentals. Hence, when $\mathbf{C}=\mathbf{A}_{L}\left(\mathbf{A}_{L}^{T} \mathbf{A}_{L}\right)^{-1}$, the error is the Euclidean distance in the HVISS as in [3].

Notice that the LMMSE estimate of the transformed tristimulus values $(\widehat{\mathbf{C t}})$ is the same as the transformed value $(\mathbf{C} \hat{\mathbf{t}})$ of the tristimulus estimate (2). This is, in turn, the LMMSE estimate of tristimulus values with respect to the directions represented by $\mathbf{V C}^{T}$. We will denote the matrix of preferred directions, $\mathbf{V} \mathbf{C}^{T}$, by the matrix $\mathrm{D}$, with columns (individual preferred directions) $\mathrm{d}_{i}$.

The estimation error between transformed $s$-stimulus values is

$$
E\left[\|\mathbf{e}\|_{e}^{2}\right]=E\left[\|\mathbf{t}-\hat{\mathbf{t}}\|_{e}^{2}\right]
$$


where $\|\cdot\|_{e}$ represents the Euclidean norm. Substituting for $\mathbf{t}$ and $\hat{\mathbf{t}}$ from (1) and (2), respectively, and simplifying gives

$$
\begin{aligned}
E\left[\|\mathbf{e}\|_{e}^{2}\right]=\operatorname{Trace}\left(\mathbf{D}^{T} \mathbf{R D}-\mathbf{D}^{T} \mathbf{R} \mathbf{M}_{H}\left(\mathbf{M}_{H}^{T} \mathbf{R} \mathbf{M}_{H}+\mathbf{R}_{n}\right)^{-}\right. & \left.\times \mathbf{M}_{H}^{T} \mathbf{R D}\right) .
\end{aligned}
$$

Both matrices in (4) are positive semi-definite, and the trace of each is hence nonnegative. The first term is independent of the recording filters and depends only on the data set and the preferred directions. It is the maximum value of the error because the second term is nonnegative. The second term depends on the recording filters - the larger its value, the smaller the error. Its maximum value is the value of the first term, because the expected value of a nonnegative quantity cannot be negative.

Proceeding as in [1], [3], [16], one may obtain a normalized measure based on Euclidean distance in the transformed space. This measure is the ratio of the value of the second term in (4) to the value of the first term, and lies between zero and unity. The larger its value, the smaller the value of the error

$$
\begin{aligned}
& \chi\left(\mathbf{D}, \mathbf{R}, \mathbf{R}_{n}, \mathbf{M}_{H}\right) \\
& \quad=\frac{\operatorname{Trace} \mathbf{D}^{T} \mathbf{R} \mathbf{M}_{H}\left(\mathbf{M}_{H}^{T} \mathbf{R} \mathbf{M}_{H}+\mathbf{R}_{n}\right)^{-} \mathbf{M}_{H}^{T} \mathbf{R D}}{\operatorname{Trace}^{T} \mathbf{R D}} .
\end{aligned}
$$

The above may be reduced to Neugebauer's $Q$-factor which is a measure of the quality of a single recording filter and negligible measurement noise, and is based on the fractional energy contained in the HVISS. The $Q$-factor of $\mathrm{x}$ is defined as [2]

$$
q(\mathrm{x})=\frac{\left\|P_{V}(\mathrm{x})\right\|_{e}^{2}}{\|\mathrm{x}\|_{e}^{2}} .
$$

To evaluate a single filter $\mathbf{m}$, particularly its effectiveness as a color filter in the combination of recording illuminant, optical path and sensor, $\mathbf{H}$, and using the simplest conditions for the measure $\chi[(5)]: \mathbf{R}=\sigma^{2} \mathbf{I}, \mathbf{R}_{n}=\mathbf{0}, \mathbf{C}=\mathbf{V}\left(\mathbf{V}^{T} \mathbf{V}\right)^{-}$, i.e., $\mathbf{D}=P_{V}$, and $\mathbf{M}_{H}=\mathbf{H m}$, gives

$$
q(\mathbf{H m})=\chi\left(P_{V}, \sigma^{2} \mathbf{I}, \mathbf{0}, \mathbf{H m}\right) .
$$

The measure $\chi$ is, unfortunately, not good enough at predicting perceptual error [1]. Hence, Wolski et al. [6] and Sharma and Trussell [1] suggest the use of Kronecker products to utilize locally linear approximations to more accurate, nonlinear, perceptual error models. Their notation provides a more general analytical form than the error expressions discussed above, and its use is justified only when errors in spaces which are not linear transformations of the space spanned by $\mathbf{V}$ are required. The approach of Wolski et al. and Sharma et al. also leads to error measures that are linear in the recording filters, and hence the rest of the analysis in this paper also holds for their approaches. We show this in detail in the Appendix, but refrain from using the Kronecker product expressions necessary to do so in the main body of the paper.

In the following section, we generalize the Euclidean inner product to include weighting of different directions based on the data statistics. This provides a common, simple framework for all color spaces and data sets as we show in Section IV. While [1] provides a common framework as well, and includes measurement noise which ours does not, our explicit use of the inner product allows simple expressions linking even the most complex-looking measures to Neugebauer's $Q$-factor. Further, and perhaps more importantly, the use of the inner product is absolutely necessary to understand the importance of orthogonality in recording filter design.

\section{GENERALIZATION OF INNER PRODUCTS, ORTHOGONALITY AND PROJECTION OPERATORS}

Data dependence generally weights different directions differently in the $N$-space of reflectance functions and recording filters, and it is useful to define the following inner product which accounts for the weighting

$$
\langle\mathbf{x}, \mathbf{y}\rangle^{\prime}=\mathrm{x}^{T} \mathbf{R y} .
$$

For (7) to define an inner product, it is necessary and sufficient that $\mathbf{R}$ be positive definite. In particular, this implies that $\mathbf{R}$ be invertible. Clearly, the inner product is the Euclidean inner product and induces the Euclidean norm when $\mathbf{R}=\mathbf{I}$. Note that this inner product defines error measures in the "parent" $N$-space and not in the lower dimensional $R(\mathbf{V})$.

\section{A. Induced Norm and Projection Operator}

Consider the norm induced by this inner product

$$
\|\mathrm{x}\|^{\prime 2}=\langle\mathbf{x}, \mathbf{x}\rangle^{\prime}=\mathbf{x}^{T} \mathbf{R x} .
$$

If $P_{X}^{\prime}$ denotes the projection operator onto the space $R(\mathbf{X})$ with respect to the inner product $\langle,\rangle^{\prime}$, i.e., $P_{X}^{\prime}(\mathbf{x})$ is the vector in $R(\mathrm{X})$ closest to $\mathrm{X}$ with respect to the norm $\|\cdot\|^{\prime}$ of (8), it can be shown that,

$$
P_{X}^{\prime}=\mathbf{X}\left(\mathbf{X}^{T} \mathbf{R X}\right)^{-1} \mathbf{X}^{T} \mathbf{R} .
$$

\section{B. Generalized Q-Factor}

The generalization of inner products and projection operators induces a generalized $Q$-factor. The $Q$-factor with respect to the space $R(\mathbf{X})$ and the generalized inner product may be defined as a generalization of Neugebauer's $Q$-factor [2] [see (6)]

$$
q_{X}(\mathrm{x})=\frac{\left\|P_{X}^{\prime}(\mathrm{x})\right\|^{2}}{\|\mathrm{x}\|^{\prime 2}}
$$

Let $\left\{\mathbf{y}_{i}\right\}_{i=1}^{\omega}$ be an orthonormal basis for $R(\mathbf{X})$ with respect to the inner product in (7) [i.e., $\mathbf{Y}^{T} \mathbf{R Y}=\mathbf{I}$ or the $\mathbf{y}_{i}$ are "R-orthonormal," and $R(\mathbf{Y})=R(\mathbf{X})$, i.e., $\mathbf{Y}=\mathbf{X} \boldsymbol{\Gamma}$ for invertible $\boldsymbol{\Gamma}]$. Then, from (9), $P_{X}^{\prime}=\mathbf{Y} \mathbf{Y}^{T} \mathbf{R}$ and (10) is

$$
q_{\mathrm{X}}^{\prime}(\mathrm{x})=\frac{\sum_{i=1}^{\omega}\left\langle\mathrm{x}, \mathrm{y}_{i}\right\rangle^{\prime 2}}{\langle\mathrm{x}, \mathrm{x}\rangle^{\prime}}
$$

in terms of the generalized inner product.

\section{Generalized Inner Product Notation for Matrices}

The generalization of inner products above can be used to rewrite some useful matrix expressions

$$
\mathbf{X}^{T} \mathbf{R Y}=\left[\left\langle\mathbf{x}_{i}, \mathbf{y}_{j}\right\rangle^{\prime}\right]
$$


where $\mathbf{x}_{i}$ and $\mathbf{y}_{i}$ are the $i$ th columns of $\mathbf{X}$ and $\mathbf{Y}$ respectively. Further

$$
\operatorname{Trace}\left(\mathbf{X}^{T} \mathbf{R Y}\right)=\sum_{i}\left\langle\mathbf{x}_{i}, \mathbf{y}_{i}\right\rangle^{\prime}
$$

\section{Generalized InNer Products AND ERror Measures}

Generalized inner products may be used to simplify the expressions for all measures based on an affine color correction procedure. The most general fidelity criterion based on a quadratic error measure for negligible measurement noise [(5)] may be simply expressed in terms of inner products as follows. Let $\mathbf{G}=\left[\mathbf{g}_{1}, \mathbf{g}_{2}, \cdots \mathbf{g}_{\gamma}\right]$ where $\left\{\mathbf{g}_{i}\right\}_{i=1}^{\gamma}$ is an $\mathbf{R}$-orthonormal basis for $R\left(\mathbf{M}_{H}\right)$. The numerator in (5) is Trace $\left(\mathbf{D}^{T} \mathbf{R G G}{ }^{T} \mathbf{R D}\right)$ and

$$
\chi\left(\mathbf{D}, \mathbf{R}, \mathbf{0}, \mathbf{M}_{H}\right)=\frac{\sum_{i=1}^{\alpha} \sum_{j=1}^{\gamma}\left\langle\mathbf{d}_{i}, \mathbf{g}_{j}\right\rangle^{\prime 2}}{\sum_{i=1}^{\alpha}\left\langle\mathbf{d}_{i}, \mathbf{d}_{i}\right\rangle^{\prime}} .
$$

One of the strengths of the measure $\nu$ was the fact that it generalized Neugebauer's $Q$-factor. An expression for $\nu$ is

$$
\nu\left(\mathbf{M}_{H}, \mathbf{V}\right)=\frac{\sum_{i=1}^{i=\beta} q\left(\mathbf{o}_{i}\right)}{\alpha}
$$

where $\left\{\mathbf{o}_{i}\right\}_{i=1}^{\beta}$ is an orthonormal basis for $R\left(\mathbf{M}_{H}\right)$ with respect to the Euclidean inner product and $\alpha$ is the dimension of $\mathrm{R}(\mathbf{V})$.

The measure $\chi$ can be represented in terms of generalized $Q$-factors as follows:

$$
\chi\left(\mathbf{D}, \mathbf{R}, \mathbf{0}, \mathbf{M}_{H}\right)=\frac{\sum_{i=1}^{\alpha} \eta_{i} q_{M_{H}}^{\prime}\left(\mathbf{d}_{i}\right)}{\sum_{i=1}^{\alpha} \eta_{i}}
$$

where $\eta_{i}$ is the energy in the $i$ th preferred direction in $R(\mathbf{V})$, $\mathrm{d}_{i}$. Thus the most general quadratic error measure consists of a weighted average of the generalized $Q$-factors of preferred directions in the target space.

Recall that $\nu$ is a special case of $\chi$ where the preferred directions are orthonormal directions in the target space. The above expression for $\chi$ easily reduces to the similar one for $\nu$ when the preferred directions are orthogonal, and the weighting of each direction is equal, or $\eta_{i}$ are all equal to unity.

The error measures of Wolski et al. [6] and Sharma et al. [1] may also be expressed in terms of inner products with some modifications. The expressions are derived in the Appendix. In the next section we illustrate the use of the generalized inner product in noise analysis.

\section{Noise Analysis Using Generalized InNER PRoducts}

The analysis performed so far ignores noise and hence implies that any set of filters $\mathbf{M}$ which maximizes expression (5) is a "good" set of filters. However, it is clear that the correction (2) may unduly amplify measurement noise, especially when the inverse problem is ill-conditioned. It is well-known that the inclusion of noise statistics in any LMMSE makes the inverse problem better-conditioned. We address the problem of conditioning in this section and derive filter design criteria to reduce noise amplification by the correction matrix. Vrhel and Trussell have addressed this problem while analyzing robustness of color correction to errors in filter design [4] and while addressing optimality of filters with respect to noise performance [8]. One of the solutions we present is similar to their solution, and is presented here in terms of inner products and orthogonality.

Here, we think of noise as that component of the output that has considerable variation over patches, and has its origin in measurement noise. The error in color reproduction which would be constant across a patch is not thought of as noise. Another way of thinking about the noise is thinking of it as the variation of the error in color reproduction, while ignoring the mean.

\section{A. Worst-Case Signal to Noise Ratio}

The min-max method of [4] may be used to analyze the worst-case signal to noise ratio (SNR) as follows. For a specific reflective spectrum $\mathbf{f}$ and noise $\mathbf{n}$, the SNR after correction is lowest when $\mathbf{M}_{H}^{T} \mathbf{f}$ is an eigenvector of minimum eigenvalue of the correction matrix (3), and $\mathbf{n}$ is an eigenvector of maximum eigenvalue. This minimum SNR is

$$
\begin{aligned}
\mathrm{SNR}_{\min } & =\left(\frac{\rho_{\min }}{\rho_{\max }}\right)^{2} \times \frac{\operatorname{Trace}\left(\mathbf{M}_{H}^{T} \mathrm{ff}^{T} \mathbf{M}_{H}\right)}{\operatorname{Trace}\left(\mathbf{n n}^{T}\right)} \\
& =\left(\frac{\rho_{\min }}{\rho_{\max }}\right)^{2} \times \epsilon=\frac{\epsilon}{\omega^{2}}
\end{aligned}
$$

where $\rho_{\min }$ and $\rho_{\max }$ are minimum and maximum eigenvalues, respectively, of the correction matrix, $\epsilon$ is the SNR before correction, and $\omega$ is the condition number of the correction matrix (the ratio of maximum to minimum eigenvalue). A max-min approach of maximizing the minimum SNR leads to maximizing $\rho_{\min } / \rho_{\max }$ or minimizing $\omega$. The optimal solution is when $\omega$ is unity, or the correction matrix is a multiple of the identity (the recording filters are identical scalar multiples of the preferred directions).

The color correction matrix, and hence its condition number, depends on the viewing illuminant $\mathbf{L}$ and the noise correlation matrix $\mathbf{R}_{n}$. While the viewing illuminant may be measured very accurately, $\mathbf{R}_{n}$ is estimated. A good assumption commonly made is that the noise consists of uncorrelated, independent, isotropically distributed variables, and $\mathbf{R}_{n}$ is usually estimated as $\sigma^{2} \mathbf{I}$, where $\sigma^{2}$ is the estimated noise variance.

When the correction matrix is not a multiple of the identity, $\omega$ is a measure of the amount of noise amplification for a specific viewing illuminant $\mathbf{L}$ and a specific estimated value of $\mathbf{R}_{n}$. It is well known in the signal/image processing community that the condition number of a transformation has a strong bearing on the noise amplification properties of a system or a method. Thus, the above result is not surprising at all. What is interesting is that the condition number of the color correction matrix is not 
always easily calculated or fixed and does not always predict image quality.

As in the image restoration problem, the estimated noise variance may be increased on purpose to better condition the color correction matrix - an increase in the estimated noise variance decreases $\omega$ and hence also the noise amplification. This, however, is at the cost of color saturation [20]. Hence $\omega$ by itself is not an accurate predictor of the image quality from a specific filter set, though it is a predictor of noise amplification.

$\omega$ (and hence the noise amplification) increases with a decrease in the estimated noise variance. Hence, calculating the value of $\omega$ as the estimated noise variance tends to zero, or for $\mathbf{R}_{n} \rightarrow \mathbf{0}$, is a means of estimating the worst-case noise amplification or the noise amplification and color saturation trade-off for a fixed filter set independent of measurement noise. A large value of $\omega$ for zero-estimated noise variance implies a basically ill-conditioned situation to start with-independent of measurement noise, of estimated noise variance, and of fixes to the noise estimates. It is useful when the filters are to be evaluated for a fixed viewing illuminant. This is not always the case (digital camera and scanner output images may need to be rendered for many different viewing conditions). In the following section we show how we can get around the limitation of a fixed viewing illuminant.

\section{B. Ratio of Expected Signal Power to Expected Noise Power}

In the previous section, we noted the effect of the color correction matrix on the worst-case noise amplification. For a given filter set, the color correction matrix-and hence the value of $\omega$-can be changed by changing the noise estimate or the viewing illuminant. In this section, we investigate other possibilities for predicting noise amplification.

The min-max method of [4] may be used to analyze the ratio of expected signal power to expected noise power after correction (denoted $S_{\exp } N_{\exp } R$ ). This analysis demonstrates why orthogonality is an important criterion in color recording filter design. The value of $S_{\exp } N_{\exp } R$ assuming signal-independent and signal-uncorrelated noise is

$$
\begin{aligned}
S_{\exp } N_{\exp } R & =\frac{E\left[\operatorname{Trace}\left(\mathbf{B} \mathbf{M}_{H}^{T} \mathrm{ff}^{T} \mathbf{M}_{H} \mathbf{B}^{T}\right)\right]}{E\left[\operatorname{Trace}\left(\mathbf{B n n}^{T} \mathbf{B}^{T}\right)\right]} \\
& =\frac{\operatorname{Trace}\left(\mathbf{B} \mathbf{M}_{H}^{T} \mathbf{R M}_{H} \mathbf{B}^{T}\right)}{\operatorname{Trace}\left(\mathbf{B} \mathbf{R}_{n} \mathbf{B}^{T}\right)}
\end{aligned}
$$

where $E[\cdot]$ represents the expectation operator.

Bounds on the value of $S_{\text {exp }} N_{\text {exp }} R$ may be obtained from the theory of matrix inequalities, as follows. Equation (11) may be rewritten as

$$
S_{\exp } N_{\exp } R=\frac{\operatorname{Trace}\left(\mathbf{X}^{T} \mathbf{Y X}\right)}{\operatorname{Trace}\left(\mathbf{X}^{T} \mathbf{X}\right)}=\frac{\sum_{i=1}^{i=s} \mathbf{x}_{i}^{T} \mathbf{Y} \mathbf{x}_{i}}{\sum_{i=1}^{i=s} \mathbf{x}_{i}^{T} \mathbf{x}_{i}}
$$

where $\mathbf{X}=\mathbf{R}_{n}^{1 / 2} \mathbf{B}^{T}$, the $\mathbf{x}_{i}$ are columns of $\mathbf{X}$, and $\mathbf{Y}=\mathbf{R}_{n}^{-(1 / 2)}\left(\mathbf{M}_{H}^{T} \mathbf{R} \mathbf{M}_{\mathbf{H}}\right) \mathbf{R}_{n}^{-(1 / 2)}$. Values of $\mathbf{x}_{i}^{T} \mathbf{Y} \mathbf{x}_{i}$ normal- ized by $\mathrm{x}_{i}^{T} \mathrm{x}_{i}$ are bounded above and below by the maximum and minimum eigenvalues of $\mathbf{Y}$ respectively, and hence so is the value of $S_{\exp } N_{\exp } R$

$$
\lambda_{\min } \leq S_{\exp } N_{\exp } R \leq \lambda_{\max }
$$

where $\lambda_{\min }$ and $\lambda_{\max }$ are minimum and maximum eigenvalues respectively of $\mathbf{R}_{n}^{-(1 / 2)}\left(\mathbf{M}_{H}^{T} \mathbf{R} \mathbf{M}_{H}\right) \mathbf{R}_{n}^{-(1 / 2)}$.

With no more knowledge about the nature of the individual matrices, an optimal solution is one where $\lambda_{\min }=\lambda_{\max }$ or, equivalently

$$
\mathbf{R}_{n}^{-(1 / 2)}\left(\mathbf{M}_{H}^{T} \mathbf{R} \mathbf{M}_{H}\right) \mathbf{R}_{n}^{-(1 / 2)}=c \mathbf{I}
$$

for a constant $c$. This implies that

$$
\mathbf{M}_{H}^{T} \mathbf{R} \mathbf{M}_{H}=c \mathbf{R}_{n}
$$

or

$$
\left\langle\mathbf{M}_{H i}, \mathbf{M}_{H j}\right\rangle^{\prime}=c E\left(\mathbf{n}_{i} \mathbf{n}_{j}\right) .
$$

Hence, the correlation matrix of optimal effective recording filters is a scalar multiple of the noise correlation matrix. This is an important result, and in this form is independent of the specific distribution of the noise variables, as long as they are signal-independent and uncorrelated with the signal.

In particular, from (13), orthogonal noise variables imply R-orthogonal optimal effective recording filters; orthogonal, isotropically distributed noise variables imply $\mathbf{R}$-orthogonal optimal effective recording filters of equal norm (which may be thought of as $\mathbf{R}$-orthonormal recording filters). The first known use of orthogonality in color recording filters appears to be in [21], though to date there has been no literature on reasons why orthogonality is important. If the noise variables are not orthogonal, i.e., there is correlation among the noise variables, optimal recording filters are similarly correlated.

In the rest of this paper, we assume that the noise variables are orthogonal and isotropically distributed, i.e., $\mathbf{R}_{n}=\sigma^{2} \mathbf{I}$, which implies that each eigenvalue $\lambda$ of $\mathbf{R}_{n}^{-(1 / 2)}\left(\mathbf{M}_{H}^{T} \mathbf{R M}_{H}\right) \mathbf{R}_{n}^{-(1 / 2)}$ is the ratio

$$
\lambda=\frac{\zeta}{\sigma^{2}}
$$

where $\zeta$ is an eigenvalue of $\mathbf{M}_{H}^{T} \mathbf{R} \mathbf{M}_{H}$. Inequality (12) becomes

$$
\zeta_{\min } \leq \sigma^{2} \times S_{\exp } N_{\exp } R \leq \zeta_{\max }
$$

The ratio of expected signal power to expected noise power before color correction is

$$
\frac{\operatorname{Trace}\left(\mathbf{M}_{H}^{T} \mathbf{R} \mathbf{M}_{H}\right)}{\operatorname{Trace}\left(\mathbf{R}_{n}\right)}=\frac{\operatorname{Trace}\left(\mathbf{M}_{H}^{T} \mathbf{R} \mathbf{M}_{H}\right)}{r \times \sigma^{2}}=\frac{1}{r \times \sigma^{2}} \sum \zeta_{i}
$$

where $r$ is the number of recording filters, or the number of measurements. This implies that the factor by which the ratio of expected signal to expected noise changes after color correction is

$$
\varsigma \equiv \frac{S_{\exp } N_{\exp } R}{\frac{1}{r \times \sigma^{2}} \sum \zeta_{i}}=\frac{\sigma^{2} \times S_{\exp } N_{\exp } R}{\frac{1}{r} \sum \zeta_{i}} .
$$



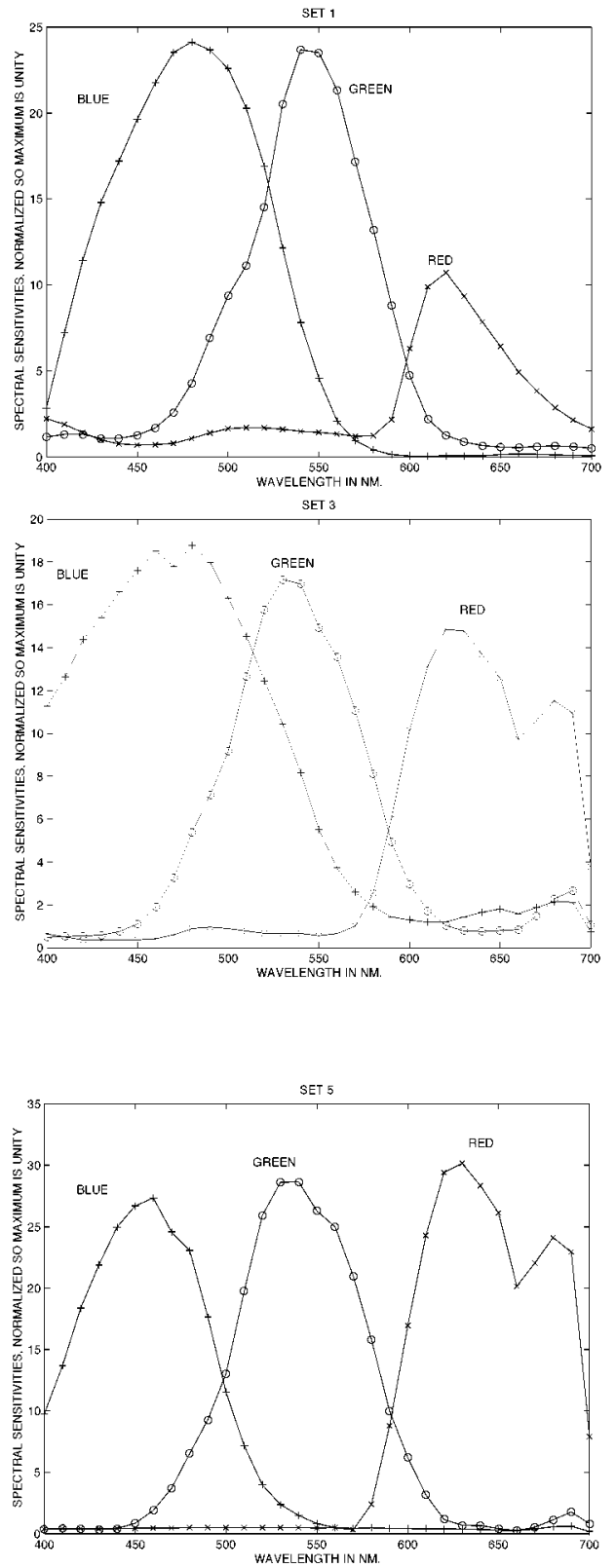
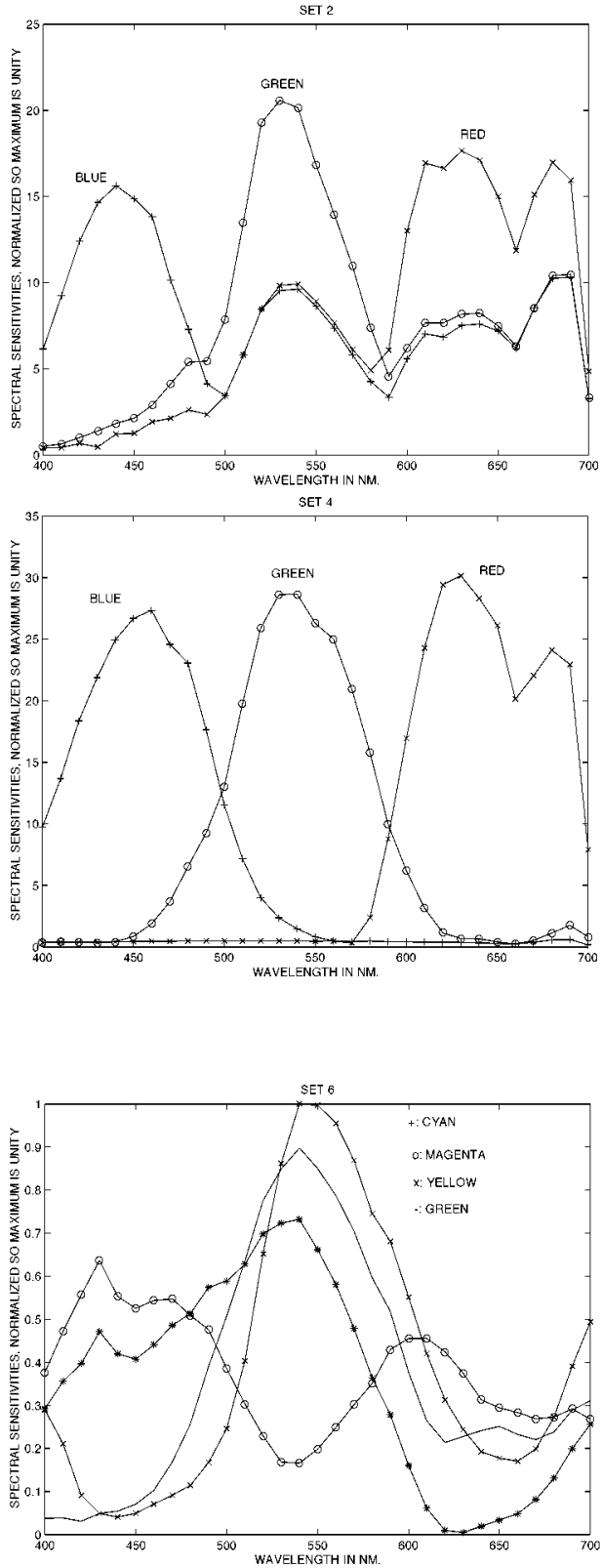

Fig. 1. Sets 1-6.

Dividing inequality (14) by the average value of $\zeta_{i}$

$$
\frac{\zeta_{\min }}{\frac{\sum \zeta_{i}}{r}} \leq \varsigma \equiv \frac{\sigma^{2} \times S_{\exp } N_{\exp } R}{\frac{\sum \zeta_{i}}{r}} \leq \frac{\zeta_{\max }}{\frac{\sum \zeta_{i}}{r}} .
$$

Using the simple algebraic inequality that the maximum and minimum values of a finite set of numbers are upper and lower bounds respectively of its average value

$$
\zeta_{\min } \leq \frac{1}{r} \sum \zeta_{i} \leq \zeta_{\max }
$$

we obtain bounds on $\varsigma$

$$
\frac{\zeta_{\min }}{\zeta_{\max }} \leq \frac{\zeta_{\min }}{\sum_{r} \zeta_{i}} \leq \varsigma \leq \frac{\zeta_{\max }}{\sum_{r} \zeta_{i}} \leq \frac{\zeta_{\max }}{\zeta_{\min }}
$$

The condition number of $\mathbf{M}_{H}^{T} \mathbf{R} \mathbf{M}_{H}$ and its inverse provide upper and lower bounds, respectively, on $\varsigma$. We refer to the condition number of $\mathbf{M}_{H}^{T} \mathbf{R} \mathbf{M}_{H}$ as $\kappa$ in the rest of this paper, and propose its use as a measure of filter orthogonality and noise sensitivity. A perfect value of $\kappa$ is unity, and indicates an R-orthonormal set of color filters. Larger values of $\kappa$ indicate "less orthonormal" filters and larger noise amplification in general.

The condition number of the color correction matrix (3), $\omega$, is distinct from the value $\kappa$, and takes the viewing illuminant and the value of $\sigma$ into consideration while evaluating the filters. An optimal value of the color correction matrix corresponds to effective recording filters that are a scalar multiple of the preferred directions, which need not be $\mathbf{R}$-orthogonal. When there are many different viewing illuminants and hence no fixed set of preferred directions, however, orthogonality is a useful criterion for optimality. The value $\kappa$ is to be used in cases where the set of illuminants is not known. 

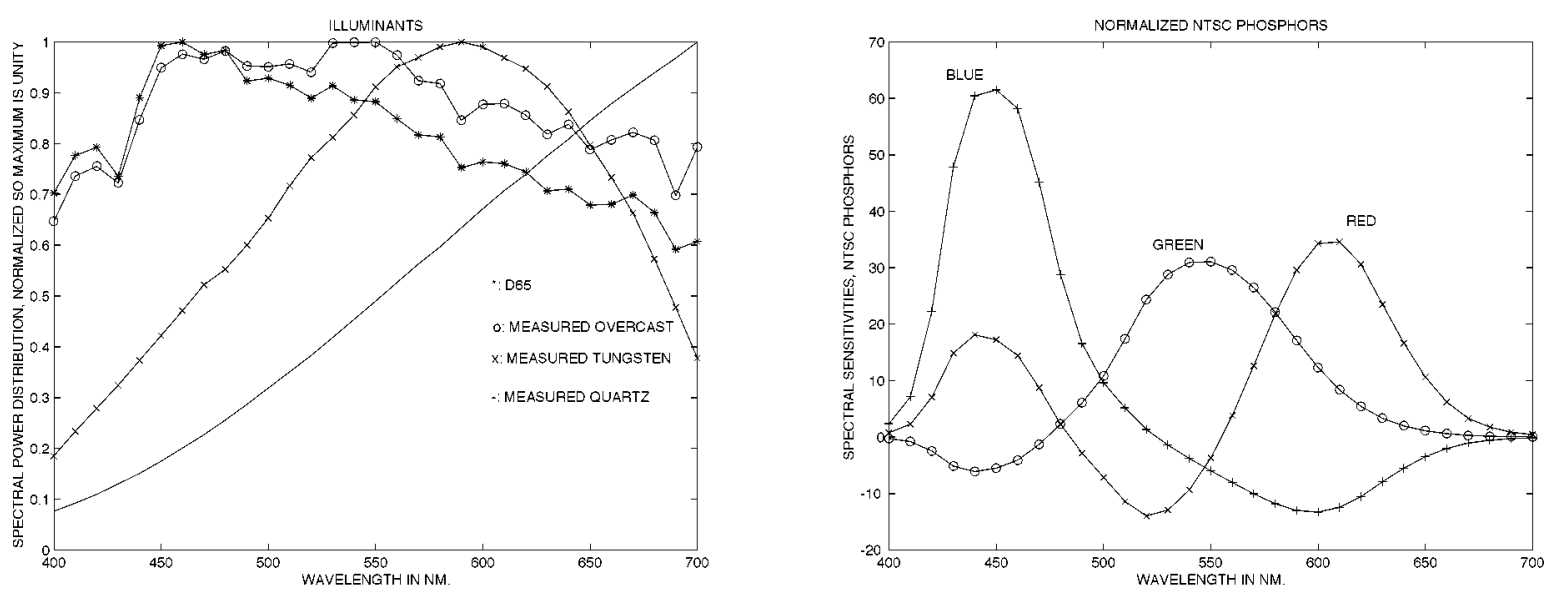

Fig. 2. Left: the illuminants; right: the matching functions corresponding to NTSC phosphors.

To recapitulate, when noise variables are signal-independent, uncorrelated and isotropic, orthonormality is important for two reasons: 1) orthonormal filters are the min-max solutions optimizing the ratio of expected signal power to expected noise power after color correction and 2) orthonormal filters are min-max solutions optimizing the amplification of the SNR after color correction. $\kappa$, the condition number of the matrix $\mathbf{M}_{H}^{T} \mathbf{R} \mathbf{M}_{H}$, is proposed as a measure of filter orthonormality.

\section{Combination of Two Optimality Criteria}

"Most optimal" color recording filters with respect to both color fidelity and noise sensitivity are those that lie along the preferred directions. If it is not possible to design filters that lie along the preferred directions (for example, it is not possible to design filters that mimic the NTSC phosphor matching functions (Fig. 8) because they have negative values at some points), and in cases where the recording filters are to be designed for a number of different viewing conditions, the "most optimal" recording filters are those that span most of the space $R(\mathbf{V})$ and are $\mathbf{R}$-orthonormal, assuming noise variables are independent among themselves and of the signal, uncorrelated among themselves and with the signal, and isotropic.

\section{EXPERIMENTAL RESULTS}

The sets of spectral responses used here are representative of several that were used in experiments during this work. We used six sets of spectral responses that are used in the manufacture of consumer digital cameras. Fig. 1 shows the normalized spectral responses. Sets 1-5 are spectral responses of sensor arrays with RGB (Red, Green, Blue) filters, while Set 6 is the response of a sensor array with cyan, magenta, yellow, green (CMYG) filters. We used four different illuminants-D65, a measured overcast daylight, a measured tungsten illuminant and a measured quartz illuminant. The normalized illuminants are plotted in Fig. 2. The preferred target directions are NTSC phosphors [22] normalized so a flat spectrum gives equal $\mathrm{R}, \mathrm{G}$, and $\mathrm{B}$ values, in the corresponding illuminant. The matching functions for the NTSC phosphors are also plotted in Fig. 2.

To test if our orthonormality measure was a valid predictor of noise performance, we performed the following simulations.
For each set, combined with each illuminant, we simulated the exact recorded values for the Vrhel-Gershon-Iwan set [23] and added zero-mean, uniformly distributed noise with variance equal to $(1 / 256 \times \text { signal maximum })^{2}$. We performed this simulation for 20 different noise sequences for each recording illuminant, thus obtaining 20 simulated measurements for each Vrhel-Gershon-Iwan data point for each filter set in each recording illuminant.

We then calculated the estimated tristimulus values for each measurement for each illuminant as viewing illuminant, assuming we knew the data-correlation matrix exactly. We calculated four different color correction matrices for each combination of recording and viewing illuminants and filter sets, assuming four different estimated values of the noise variance (zero, estimated variance equal to the actual simulated noise variance, estimated noise variance equal to twice and ten times the noise variance) and uncorrelated, independent, identically distributed noise variables, i.e., $\mathbf{R}_{n}=\mathbf{0} ; \mathbf{R}_{n}=k \mathbf{I}$; $\mathbf{R}_{n}=2 k \mathbf{I}$; and $\mathbf{R}_{n}=10 k \mathbf{I}$ where $k$ is the actual simulated noise variance. Thus, for each measurement, we estimated 16 different tristimulus vectors corresponding to four different viewing illuminants and four different estimates of $\mathbf{R}_{n}$.

We then calculated the value of $\varsigma[(15)]$ for each combination of viewing illuminant, recording illuminant, noise estimate and filter set (384 values in all). We examined the values of different criteria considered in this paper (the condition number, $\omega$, of the color correction matrix $\mathbf{B}$; and the condition number, $\kappa$, of the matrix $\mathbf{M}_{H}^{T} \mathbf{R} \mathbf{M}_{H}$ ) to determine their efficacy as predictors of $\varsigma$, the factor by which the ratio of expected signal to expected noise changes after color correction. We present plots with the data values on the $y$-axis vs. predictors on the $x$-axis in the following figures.

Fig. 3 shows a scatter plot of the value of $\varsigma$ vs. $\omega$, the condition number of the corresponding color correction matrix. Both $\omega$ and $\varsigma$ depend on the viewing illuminant as well as the estimated value of $\mathbf{R}_{n}$. $\omega$ predicts the value of $\varsigma$ well, as expected.

Increasing the value of the estimated noise variance decreases the condition number $\omega[20]$ and hence increases $\varsigma$, but it decreases color saturation [20]. As described in Section V-A, it is useful to look at the value of $\omega$ as the noise variance tends to zero to get an estimate of the basic noise amplification prop- 

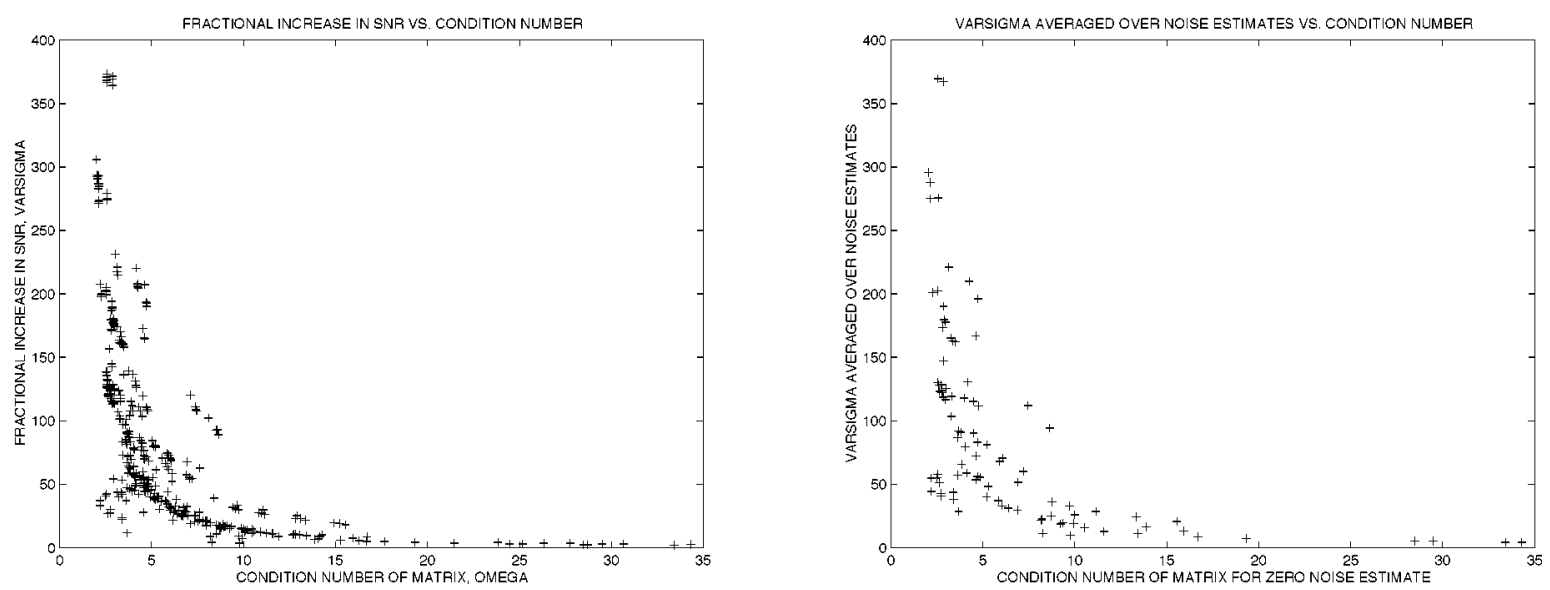

Fig. 3. Left: $\varsigma$ versus $\omega$; right: average $\varsigma$ over different noise estimates versus $\omega$, noise estimate $=0$.

erties of the color correction matrix-independent of measurement noise, noise estimates, and fixes to decrease noise amplification which also decrease color saturation. Fig. 3 also shows a scatter plot of the average value of $\varsigma$ over the different estimated noise variances versus $\omega$ with estimated value of variance equal to zero. The plot is similar to the previous one, and average values of $\varsigma$ are well-predicted by values of $\omega$ for zero estimated noise variance.

The value of $\omega$ for zero estimated noise variance, though independent of noise variance, requires knowledge of the viewing and recording illuminants. It would be useful to observe the ability of the condition number of the color correction matrix to predict noise amplification when only one illuminant, or neither illuminant, is known. We present two plots, Figs. 4 and 5, that fix the viewing and recording illuminants, respectively.

Fig. 4 shows the scatter plot of the average value of $\varsigma$ over the different estimated noise variances and the four different recording illuminants while fixing the viewing illuminant vs. the condition number of the color correction matrix assuming zero noise variance and assuming that the recording illuminant is identical to the viewing illuminant. Fig. 5 shows a scatter plot of the average value of $\varsigma$ over the different estimated noise variances and the four different viewing illuminants while fixing the recording illuminant vs. the same values.

Figs. 4 and 5 show very similar graphs. While the value of $\varsigma$ decreases in general with respect to the value of $\omega$, it is not monotonic, and the value of $\omega$ does not seem to predict well the value of $\varsigma$ when averaged over many different viewing or recording illuminants. The data points for Set 6 are plotted as asterisks $(*)$ and they lie well in the middle of the plots. Hence, as described in Section V-A, it is necessary to use predictors other than the condition number of the color correction matrix when the viewing and recording illuminants are not fixed or not known.

Figs. 6 and 7 show the same data as in Fig. 5 versus the natural logarithm of the value of $\kappa$, for $E[\mathrm{ff}]^{T}=\mathbf{I}$ and $E[\mathrm{ff}]^{T}=\mathbf{R}$, respectively. The values of $\kappa$ are measures of filter orthonormality using two different inner products: $E[\mathrm{ff}]^{T}=\mathbf{I}$ corresponds to the Euclidean inner product and $E[\mathrm{ff}]^{T}=\mathbf{R}$ to the data-weighted inner product. Fig. 7 shows a clear monotonic decreasing relationship between $\varsigma$ averaged over many viewing

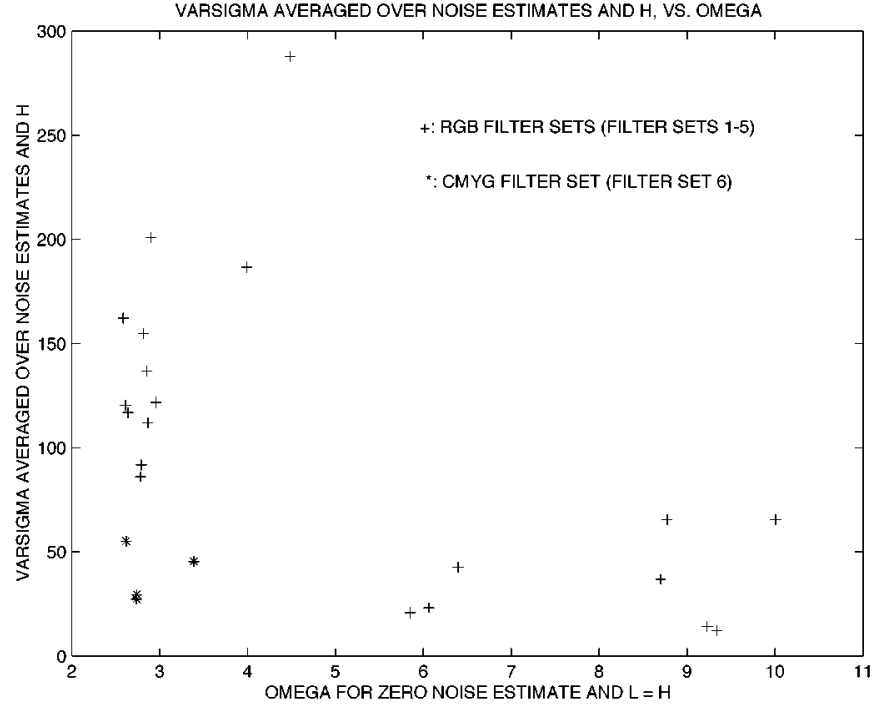

Fig. 4. $\varsigma$ averaged over noise estimates and different recording illuminants, versus $\omega$, viewing and recording illuminants identical.

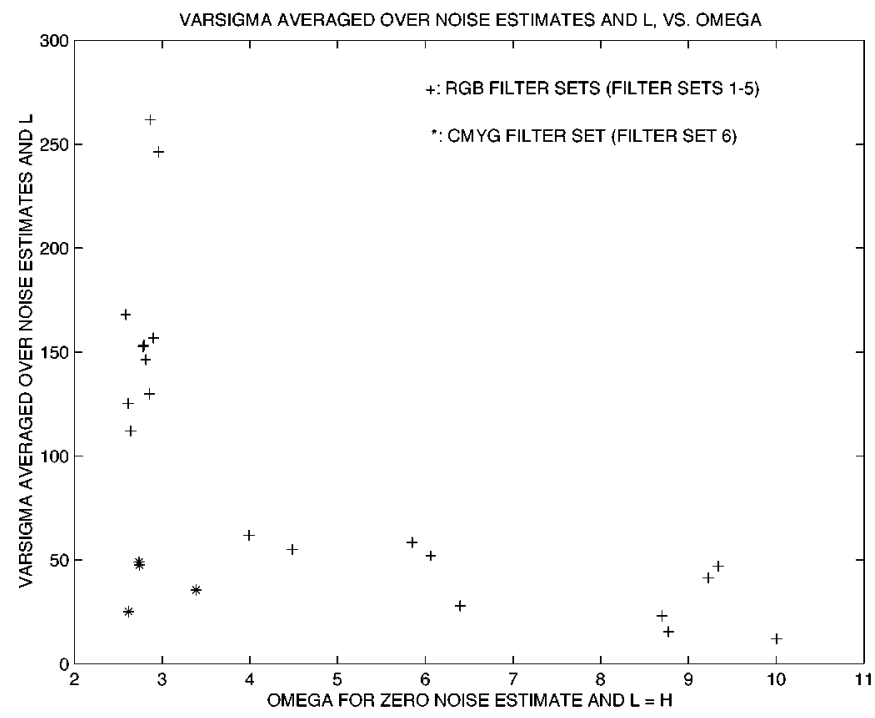

Fig. 5. $\varsigma$ averaged over noise estimates and different viewing illuminants, versus $\omega$, viewing and recording illuminants identical. 


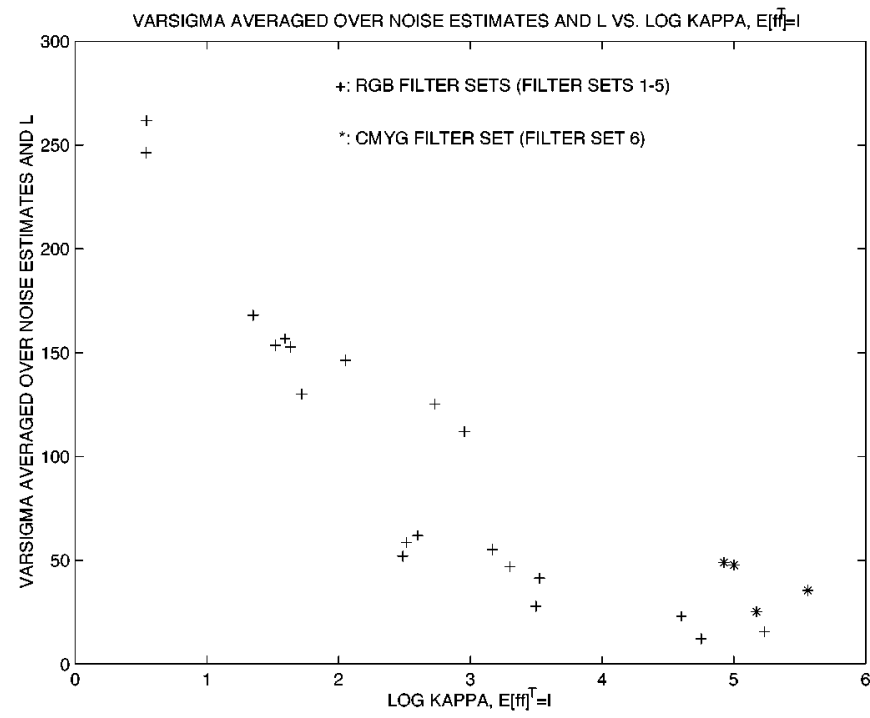

Fig. 6. $\varsigma$ averaged over noise estimates and different viewing illuminants versus $\kappa$ for $E \mathbf{f f}^{T}=\mathbf{I}$.

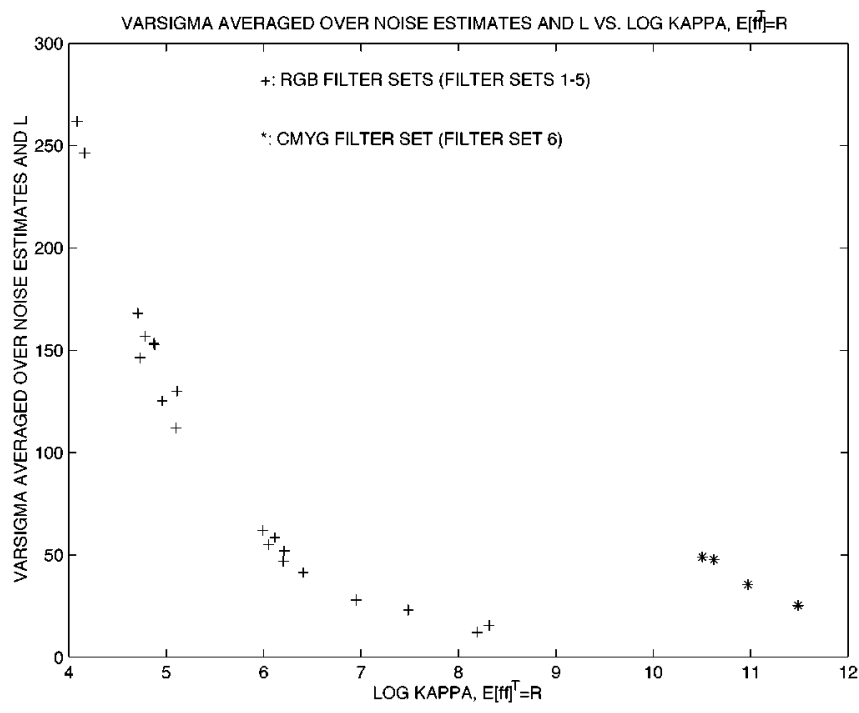

Fig. 7. $\varsigma$ averaged over noise estimates and different viewing illuminants versus $\kappa$ for $E \mathbf{f f}^{T}=\mathbf{R}$.

illuminants and the value of $\kappa$ for $E[\mathrm{ff}]^{T}=\mathbf{R}$. The points that do not fit as well in this graph as most of the points are points associated with Set 6, and are shown with asterisks (*) as in Figs. 4 and 5 .

Figs. 6 and 7 demonstrate quite clearly that $\kappa$, a measure of filter orthonormality, is at least as good a measure of noise sensitivity as $\omega$, though it depends upon fewer variables (it is independent of the viewing illuminant and of the estimated noise variance).

Figs. 8 and 9 show the average of $\varsigma$ over the different noise estimates as well as over both recording and viewing illuminants, versus the natural logarithm $\kappa$ for $E[\mathrm{ff}]^{T}=\mathbf{I}$ and $E[\mathrm{ff}]^{T}=\mathbf{R}$ respectively. The values of $\kappa$ in this case are different from those in Figs. 6 and 7 because they have been calculated for $\mathbf{H}=\mathbf{I}$. $\kappa$ is clearly a good predictor of average performance over different viewing and recording illuminants, especially when the data set is known. The sets with high values of $\kappa$ either have high overlap

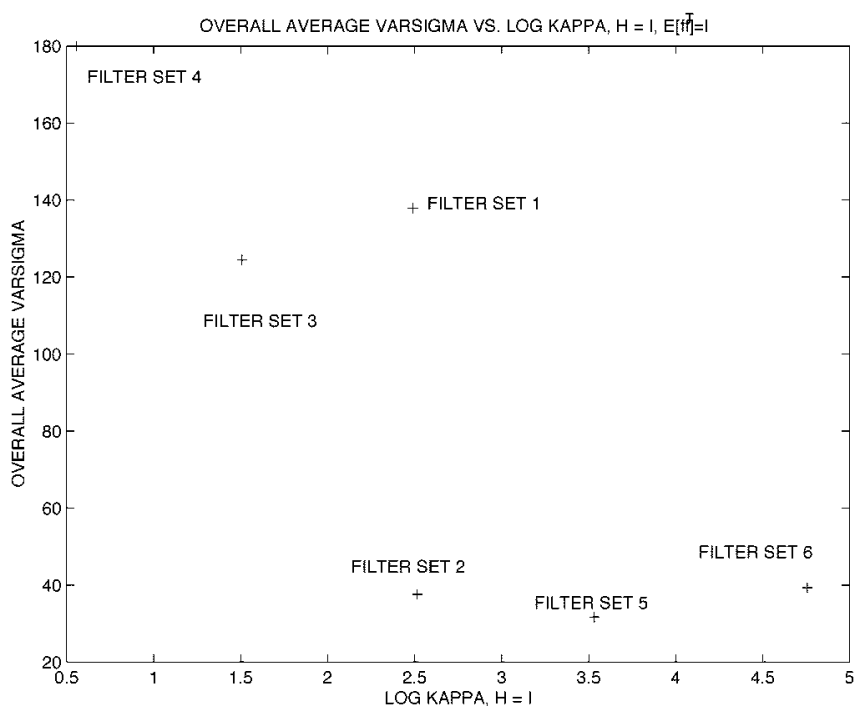

Fig. 8. Average $\varsigma$ over all conditions versus orthonormality criterion without recording illuminant, $E \mathbf{f f}^{T}=\mathbf{I}$.

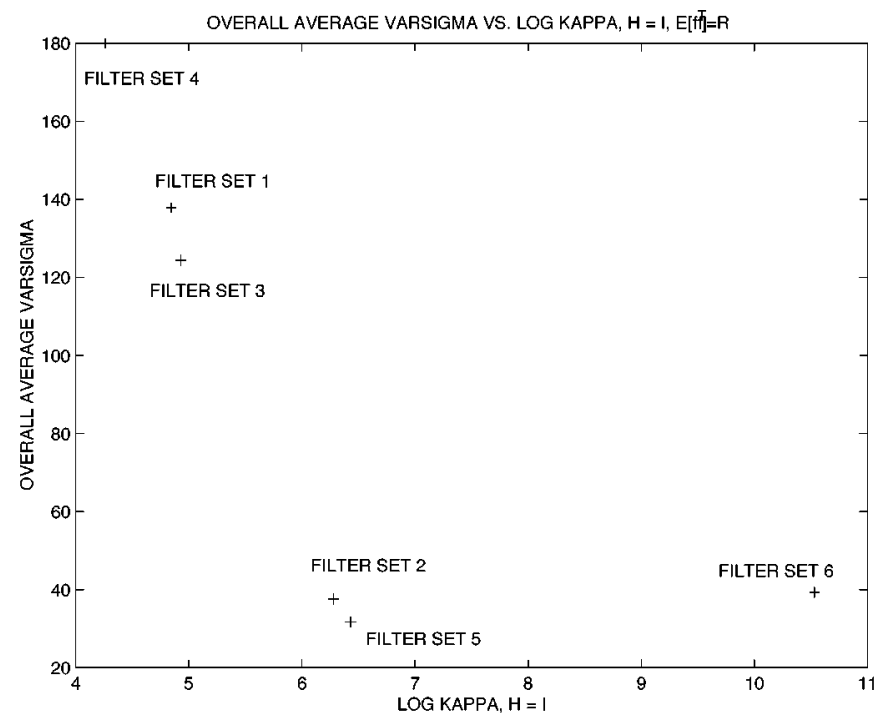

Fig. 9. Average $\varsigma$ over all conditions versus orthonormality criterion without recording illuminant, $E \mathbf{f f}^{T}=\mathbf{R}$.

among the spectral response functions of each channel, (Sets 2 and 6) or have very different channel gains as well as fairly high overlap (Set 5) and are hence far from orthonormal.

\section{CONCLUSIONS}

We have built on the linear model ideas of [2], [13]-[15], [11], [3] to demonstrate how the use of inner products and orthogonality can simplify the procedure of color recording filter design. We have shown how definitions of inner products and orthogonality are influenced by the choice of error measures for color recording filters, and we have used these ideas to develop a generalized $Q$-factor as an extension of Neugebauer's $Q$-factor [2]. We used these ideas to show that optimal recording filters with respect to noise sensitivity are orthonormal filters when the noise variables are orthogonal and isotropically distributed. Lastly, we have presented simulations to support our claims. 


\section{APPENDIX}

\section{A. Accurate Approximations of Perceptual Error}

The average $\Delta E_{a b}$ error in CIE $L^{*} a^{*} b^{*}$ space [12,pg. 166] is a perceptual error which is not equivalent to Euclidean distance in any linear transformation of CIE tristimulus space. This motivates the analysis of nonquadratic error measures. If $\mathcal{F}(\mathbf{t}(\mathbf{f}))$ and $\mathcal{F}(\hat{\mathbf{t}}(\mathbf{f}))$ represent the nonlinear transformation of real and estimated $s$-stimulus values respectively, to a transformed space where the Euclidean distance represents a valid error measure, then the error measure is $\|\mathcal{F}(\mathbf{t}(\mathbf{f}))-\mathcal{F}(\hat{\mathbf{t}}(\mathbf{f}))\|^{2}$. A locally linear approximation of $L^{*} a^{*} b^{*}$ error proposed by Wolski et al. [6] allows the use of linear models to analyze nonquadratic error measures as follows.

If the error between estimated and real $s$-stimulus values is small, and the transformation $\mathcal{F}$ is differentiable with continuous first partial derivatives at both points, the error measure can be approximated by the first term of the Taylor series approximation [1], [6]. The linear transformation $\mathbf{B}$ that minimizes the approximated error measure is defined by [1]

$$
\begin{aligned}
\operatorname{vec} \mathbf{B}= & {\left[\left(\mathbf{M}_{H}^{T} \otimes \mathbf{I}_{r}\right) \mathbf{S}_{f}\left(\mathbf{M}_{H} \otimes \mathbf{I}_{r}\right)+\mathbf{S}_{n}\right]^{-1} } \\
& \cdot\left(\mathbf{M}_{H}^{T} \otimes \mathbf{I}_{r}\right) \mathbf{S}_{f} \operatorname{vec}\left(\mathbf{A}_{L}^{T}\right)
\end{aligned}
$$

where

$$
\begin{aligned}
\mathbf{S}_{f} & =E\left\{\left(\mathrm{ff}^{T} \otimes\left(J_{\mathcal{F}}^{T}(\mathbf{t}(\mathbf{f}))\right)\right\}\right. \\
\mathbf{S}_{n} & =\mathbf{R}_{n} \otimes E\left\{\left(J_{\mathcal{F}}^{T}(\mathbf{t}(\mathbf{f}))\right)\right\} .
\end{aligned}
$$

As in (5), we obtain a normalized fidelity measure which depends on both the preferred directions in the target space as well as the transformation from the target space to a perceptually uniform space as shown in (17) at the bottom of the page. The main difference between expressions (5) and (17) is that the latter treats the entire matrix $\mathrm{D}$ as one vector in $s \mathrm{~N}$-space, unlike the former which treats each column of D, i.e., each preferred direction, as a separate vector. Further, the latter treats each column of $\mathbf{M}_{H}$ as a vector in $r \mathrm{~N}$-space, with zeros padding the $\mathrm{N}$-vector

Because of these differences, (17) allows the use of product terms of the form $\sum w_{i j k l} \mathbf{m}_{l}(i) \mathbf{m}_{k}(j)$ (elements of the matrix $\left.\mathbf{M}_{H}^{T} \otimes \mathbf{I}_{r}\right) \mathbf{S}_{f}\left(\mathbf{M}_{H} \otimes \mathbf{I}_{r}\right)$ ) while (5) allows only terms of the form $\sum w_{i j} \mathbf{m}_{l}(i) \mathbf{m}_{k}(j)$ (elements of the matrix $\mathbf{M}_{H}^{T} \mathbf{R} \mathbf{M}_{H}$ ). In other words, (17) allows the use of different weights for each product term, $\mathbf{m}_{l}(i) \mathbf{m}_{k}(j)$, while (5) requires the same weighting factors for fixed wavelength positions-in (5) the weights depend on only $i$ and $j$.

Another difference is that the weights (elements of the matrices $\mathbf{R}$ and $\mathbf{S}_{f}$ for the former and latter respectively) depend only on the data and not on $\mathbf{M}_{H}$ or $\mathbf{V}$ in the former case, but depend on both the data and $\mathbf{V}$ in the latter. However, the two

expressions are similar in that the weights in both do not depend on $\mathbf{M}_{H}$ and both can be expressed in terms of inner products-(17) in $r \mathrm{~N}$-space or $s \mathrm{~N}$-space, (5) in N-space.

To extend the ideas of Section IV to accurate approximations of nonlinear error measures, the definitions of inner products, projection operators and $Q$-factors need to be slightly modified. The matrix $\mathbf{D}$ is replaced by the vector vec $(\mathbf{D})$, the matrix $\mathbf{M}_{H}$ by the matrix $\mathbf{M}_{H} \otimes \mathbf{I}_{r}$, and the matrix $\mathbf{R}$ by the matrix $\mathbf{S}_{f}$. The expression of (17) for negligible noise may be represented in terms of inner products as follows. If

$$
\langle\mathbf{x}, \mathbf{y}\rangle^{\mathcal{F}}=\mathbf{x}^{T} \mathbf{S}_{f} \mathbf{y}
$$

and $\left\{\mathbf{g}_{i}\right\}_{i=1}^{\gamma}$ is an $\mathbf{S}_{f}$-orthonormal basis for $R\left(\mathbf{M}_{H} \otimes \mathbf{I}_{r}\right)$ in $r N$-space, then

$$
\chi_{\mathcal{F}}\left(\mathbf{D}, \mathbf{S}_{f}, \mathbf{0}, \mathbf{M}_{H}\right)=\frac{\sum_{j=1}^{\gamma}\left(\left\langle\operatorname{vec}(\mathbf{D}), \mathbf{g}_{j}\right\rangle^{\mathcal{F}}\right)^{2}}{\langle\operatorname{vec}(\mathbf{D}), \operatorname{vec}(\mathbf{D})\rangle^{\mathcal{F}}} .
$$

In terms of generalized $Q$-factors

$$
\chi_{\mathcal{F}}\left(\mathbf{D}, \mathbf{R}, \mathbf{0}, \mathbf{M}_{H}\right)=q_{M_{H} \otimes I_{r}}^{\mathcal{F}}(\operatorname{vec}(\mathbf{D})) .
$$

f

$\mathbf{V}=\left[\mathbf{v}_{1}, \mathbf{v}_{2}, \cdots \mathbf{v}_{s}\right]$

$\mathbf{t}=\mathbf{V}^{T} \mathbf{f}$, and $\hat{\mathbf{t}}$

$$
\begin{aligned}
& \mathbf{A}=\left[\mathbf{a}_{\mathbf{1}} \mathbf{a}_{\mathbf{2}} \mathbf{a}_{\mathbf{3}}\right] \\
& \mathbf{M}=\left[\mathbf{m}_{1} \mathbf{m}_{2} \cdots \mathbf{m}_{r}\right] \\
& \mathbf{h}
\end{aligned}
$$

1

\section{$\mathbf{H}$ and $\mathbf{L}$}

$\mathbf{M}_{H}$ and $\mathbf{A}_{L}$

\section{$\mathbf{A}_{L}^{T} \mathbf{f}$}

$\mathbf{g}=\mathbf{M}_{H}^{T} \mathbf{f}+\mathbf{n}$

B

C

\section{Notation}

Reflective spectrum.

Matrix of $s$ target measurement filters.

$s$-stimulus values of $\mathbf{f}$ and their linear minimum mean square error (LMMSE) estimates, respectively.

Matrix of the CIE matching functions.

Matrix of $r$ recording filters.

Combination of optical path, recording illuminant and sensor characteristic.

Viewing illuminant.

Diagonal matrices with $\mathbf{H}_{i i}=$ $\mathbf{h}(i)$ and $\mathbf{L}_{i i}=\mathrm{l}(i)$, respectively. Matrix products HM (or "effective recording system") and $\mathbf{L A}$, respectively.

CIE tristimulus vector of reflective spectrum $f$ under viewing illuminant $\mathbf{l}$.

Recorded noisy measurements.

Color correction matrix.

Linear transformation of $s$-stimulus values for error metric.

$$
\chi_{\mathcal{F}}\left(\mathbf{D}, \mathbf{S}_{f}, \mathbf{S}_{n}, \mathbf{M}_{H}\right)=\frac{\operatorname{vec}\left(\mathbf{D}^{T}\right)^{T} \mathbf{S}_{f}\left(\mathbf{M}_{H} \otimes \mathbf{I}_{r}\right)\left[\left(\mathbf{M}_{H}^{T} \otimes \mathbf{I}_{r}\right) \mathbf{S}_{f}\left(\mathbf{M}_{H} \otimes \mathbf{I}_{r}\right)+\mathbf{S}_{n}\right]^{-1}\left(\mathbf{M}_{H}^{T} \otimes \mathbf{I}_{r}\right) \mathbf{S}_{f} \operatorname{vec}\left(\mathbf{D}^{T}\right)}{\operatorname{vec}\left(\mathbf{D}^{T}\right)^{T} \mathbf{S}_{f} \operatorname{vec}\left(\mathbf{D}^{T}\right)}
$$


$\mathrm{D}$

$\mathbf{R}, \mathbf{R}_{n}$

$\chi(),. \chi^{\mathcal{F}}($.

$q(),. q^{\prime}(),. q^{\mathcal{F}}($.

$\eta_{i}$

$\sigma^{2}$

$\rho, \lambda$, and $\zeta$

$\kappa$ and $\omega$

$J_{\mathcal{F}}$

$R(\mathbf{X})$

$\mathrm{X}^{-}$

$\langle,\rangle_{e},\langle,\rangle^{\prime}$

$\|\cdot\|_{e},\|\cdot\|^{\prime}$,

$P_{X}, P_{X}^{\prime}$

$\operatorname{vec}(\mathbf{X})$

$\otimes$

\section{REFERENCES}

[1] G. Sharma and H. J. Trussell, "Figures of merit for color scanners and cameras," IEEE Trans. Image Processing, vol. 6, pp. 990-1001, July 1997.

[2] H. E. J. Neugebauer, "Quality factor for filters whose spectral transmittances are different from color mixture curves, and its application to color photography," J. Opt. Soc. Amer, vol. 46, pp. 821-824, Oct. 1956.

[3] P. L. Vora and H. J. Trussell, "Measure of goodness of a set of color scanning filters," J. Opt. Soc. Amer. A, vol. 10, pp. 1499-1508, July 1993.
[4] M. J. Vrhel and H. J. Trussell, "Filter considerations in color correction," IEEE Trans. Image Processing, vol. 3, pp. 147-161, Mar. 1994.

[5] H. J. Trussell, G. Sharma, P. Chen, and S. A. Rajala, "Comparison of measures of goodness of sets of color scanning filters," in Proc. IEEE 9th Multi-Dimensional Signal Processing Workshop, Belize, Mar. 1996, pp. 98-99.

[6] M. Wolski, C. A. Bouman, J. P. Allebach, and E. Walowit, "Optimization of sensor response functions for colorimetry of reflective and emissive objects," IEEE Trans. Image Processing, vol. 5, pp. 507-517, Mar. 1996.

[7] G. D. Finlayson and M. S. Drew, "The maximum ignorance assumption with positivity," in Proc. 4th Color Imaging Conf. Color, Science, Systems, Applications, 1996, IS\&T/SID, pp. 202-205.

[8] M. J. Vrhel and H. J. Trussell, "Optimal color filters in the presence of noise," IEEE Trans. Image Processing, vol. 4, June 1995.

[9] P. L. Vora and H. J. Trussell, "Mathematical methods for the analysis of color scanning filters," IEEE Trans. Image Processing, vol. 6, pp. 312-320, Feb. 1997.

[10] G. Sharma and H. Trussell, "Color scanner performance trade-offs," in Proc. SPIE Color Imaging: Device-Independent Color, Color Hard Copy, Graphic Arts, J. Bares, Ed., 1996, vol. 2658, pp. 270-278.

[11] H. J. Trussell, "Applications of set theoretic methods to color systems," Color Res. Applicat., vol. 16, pp. 31-41, Feb. 1991.

[12] G. Wyszecki and W. S. Stiles, Color Science: Concepts and Methods, Quantitative Data and Formulae. New York: Wiley, 1982.

[13] W. A. Shapiro, "Generalization of tristimulus coordinates," J. Opt. Soc. Amer., vol. 56, pp. 795-802, June 1966.

[14] J. B. Cohen and W. E. Kappauf, "Metameric color stimuli, fundamental metamers and Wyszecki's metameric blacks," Amer. J. Psychol., vol. 95, pp. 537-564, 1982.

[15] B. A. Wandell, "The synthesis and analysis of color images," IEEE Trans. Pattern Anal. Machine Intell., vol. PAMI-9, pp. 2-13, Jan. 1987.

[16] P. L. Vora, "Optimization criteria and numerical analysis in the design of color scanning filters," Ph.D. dissertation, North Carolina State Univ., Raleigh, 1993.

[17] T. S. Lomheim and L. S. Kalman, "Analytical modeling and digital simulation of scanning charge-coupled device imaging systems," in ElectroOptical Displays, M. A. Karim, Ed. New York: Marcel Dekker, 1992.

[18] P. L. Vora, J. E. Farrell, J. D. Tietz, and D. H. Brainard, "Digital color cameras-1-response models,” Hewlett-Packard Tech. Rep. HPL-97-53, Mar. 1997.

[19] P. L. Vora, M. L. Harville, J. E. Farrell, J. D. Tietz, and D. H. Brainard, "Digital image capture: Synthesis of sensor responses from multispectral images," in Proc. SPIE IS Conf. Color Imaging: Device-Independent Color, Color Hard Copy, and Graphic Arts II, vol. 3018, San Jose, CA, Feb. 10-14, 1997, pp. 2-11.

[20] P. L. Vora and C. E. Herley, "Trade-offs between noise sensitivity and color saturation in image sensors," in Proc. Int. Conf. Image Processing, Chicago, IL, Oct. 1998.

[21] D. L. MacAdam, "Dependence of color mixture functions on choice of primaries," J. Opt. Soc. Amer., vol. 43, pp. 533-538, June 1953.

[22] W. K. Pratt, Digital Image Processing, 2nd ed. New York: Wiley, 1991.

[23] M. J. Vrhel, R. Gershon, and L. Iwan, "The measurement and analysis of object reflectance spectra," Color Res. Applicat., vol. 19, pp. 4-9, Feb. 1994.

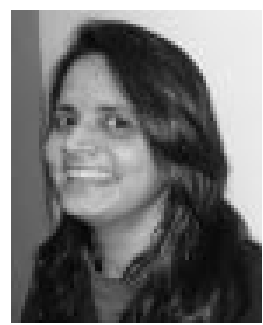

Poorvi L. Vora received the B.Tech. degree in electrical engineering from the Indian Institute of Technology, Bombay, in 1982, the M.S. degree in mathematics from Cornell University, Ithaca, NY, in 1990, the M.S. degree in electrical engineering and the Ph.D. degree in computer engineering from North Carolina State University, Raleigh, in 1988 and 1993, respectively.

She is with Hewlett-Packard Laboratories, Corvallis, OR. Her current interests are in the applications of cryptography to problems in the fields of privacy protection and digital asset commerce. 